\title{
Ankle-foot Orthosis With an Oil Damper Versus Nonarticulated Ankle-foot Orthosis in the Gait of Patients With Subacute Stroke: A Randomized Controlled Trial
}

\section{Sumiko Yamamoto ( $\nabla$ sumiko-y@iuhw.ac.jp )}

International University of Health and Welfare https://orcid.org/0000-0001-6402-873X

\section{Naoyuki Motojima}

Showa University Graduate School of Nursing and Rehabilitation Sciences School of Nursing and Rehabilitation Sciences: Showa Daigaku Daigakuin Hoken Iryogaku Kenkyuka Hoken Iryo Gakubu Yosuke Kobayashi

Nakaizu Rehabilitation Center

\section{Yuji Osada}

Tokushima Bunri University: Tokushima Bunri Daigaku

\section{Souji Tanaka}

Higashi Kanagawa Rehabilitation Hospital

\section{Aliyeh Daryabor}

Shahid Beheshti University of Medical Sciences School of Rehabilitation

\section{Research}

Keywords: Ankle-foot orthosis, stroke, resistive moment, gait, shank-to-vertical-angle, power absorption

Posted Date: April 7th, 2021

DOI: https://doi.org/10.21203/rs.3.rs-376006/v1

License: (9) This work is licensed under a Creative Commons Attribution 4.0 International License. Read Full License 
1 Ankle-Foot orthosis with an oil damper versus nonarticulated ankle-foot

2 orthosis in the gait of patients with subacute stroke: A randomized controlled trial

3

4 Authors

5 Sumiko Yamamoto, Ph.D.

6 Graduate school, International University of Health \& Welfare,

7 4-1-26, Akasaka, Minato-ku, Tokyo, 107-8402, Japan

$8 \quad$ E-mail: sumiko-y@iuhw.ac.jp

9 ORCID: $\underline{\text { http://orcid.org/0000-0001-6402-873X }}$

10

11 Naoyuki Motojima, Ph.D.

12 Showa University School of Nursing and Rehabilitation Science

13 Tohkaichibacho1865, Midoriku, Yokohama, Kanagawa 226-8555, Japan

14 E-mail: n.motojima@gmail.com

15 ORCID : $\underline{\text { https://orcid.org/0000-0001-6675-7995 }}$

16

17 Yosuke Kobayashi

18 Nakaizu Rehabilitation Center

19 1523-108, Hiekawa, Izu, Shizuoka, 410-2507, Japan

20 E-mail: yosukekopayashi@yahoo.co.jp

21

Yuji Osada, PhD

23 Department of Health and Welfare Tokushima Bunri University

24 Nishihamahoji-180, Yamashirocho, Tokushima 770-8514, Japan

25 E-mail: osada@tks.bunri-u.ac.jp

26 ORCID : https://orcid.org/0000-0003-0433-5871

27

Souji Tanaka, Ph.D.

Higashi Kanagawa Rehabilitation Hospital

30 1-13-10, Nishikanagawa, Kanagawa-ku, Kanagawa, 221-0822, Japan

31 E-mail: $\underline{\text { soji.tanaka.gs@gmail.com }}$

32

ORCID: https://orcid.org/0000-0003-4494-0240

33 
36 Aliyeh Daryabor, Ph.D.

37 School of Rehabilitation, Shahid Beheshti University of Medical Sciences

38 Velenjak St., CHamran Highway, Tehran, Iran

39 E-mail: r_daryabor@yahoo.com

40 ORCID: https://orcid.org/0000-0002-0652-6025

41

42

43

44 Corresponding author

45 Sumiko Yamamoto

46 International University of Health \& Welfare

47 4-1-26, Akasaka, Minato-ku, Tokyo, 107-8402, Japan

48 Tel: +81-5574-3874; Fax: +81-5574-3901

49 Email: sumiko-y@iuhw.ac.jp

50

51

52

53

54

55

56

57

58

59

60

61

62

63

64 
Background: Gait improvement in patients with stroke using ankle-foot orthosis (AFO) has been compared to the effects of non-AFO use in previous studies, but the effect of different kinds of AFOs has not been clear. When considering the effect of different kinds of AFOs on gait, the dorsiflexion and plantar flexion moment of resistance is considered a key determinant of functional effect. In this study, the effect on gait of using an AFO with an oil damper (AFO-OD), which has plantar flexion resistance but no dorsiflexion resistance, and a nonarticulated AFO, which has both dorsiflexion and plantar flexion resistance, were compared in a randomized controlled trial.

Methods: Forty-one patients (31 men, 10 women; mean age $58.4 \pm 11.3$ years) in the subacute phase of stroke were randomly allocated to two groups to undergo 2 weeks of gait training by physiotherapists while wearing an AFO-OD or a nonarticulated AFO. A motion capture system was utilized to measure shod gait without orthosis at baseline and after training with the allocated AFO. Data analysis was performed focused on the spatial and temporal parameters, ground reaction force, shank-to-vertical angle, and ankle joint kinematics and kinetics. Two-way mixed ANOVA was performed to clarify the effect of AFO use and the difference between the two AFOs. Results: Thirty-six patients completed the study (17 in the AFO-OD group and 19 in the nonarticulated AFO group). Spatial and temporal parameters and ankle joint kinematics were improved after 2 weeks in both AFO groups. Interactions were found for the range of shank-to-vertical angles in paretic single stance and ankle peak power absorption. In the AFO-OD group, both parameters improved when the participants walked with the AFO compared to the shod gait, but there was no change in the nonarticulated AFO group. Power generation was not increased in either AFO group. dorsiflexion resistance improved the range of the shank-to-vertical angle and ankle power absorption but not power generation in a paretic stance. 
89 (336/350 words)

90 Trial registration: UMIN000028126 Registered 1 August 2017,

91 https://upload.umin.ac.jp/cgi-bin/icdr/ctr_menu_form_reg.cgi?recptno=R000032197

92

93 Key words: Ankle-foot orthosis, stroke, resistive moment, gait, shank-to-vertical-angle,

94 power absorption

95

96 Abbreviations

97 AFO: ankle-foot orthosis

98 AFO-OD: ankle-foot orthosis with an oil damper

99 SVA: shank-to-vertical angle

100

101

102

103

104

105

106

107

108

109

110

111

112 


\section{Introduction}

114 Ankle-foot orthoses (AFOs) are commonly used in clinical practice to improve gait in patients

115 with stroke. Systematic reviews have shown that AFOs can improve stroke gait by modifying the

116 kinematics of the ankle and knee joints in the stance phase and preventing foot drop in the swing

117 phase [1,2]. However, the evidence for the use of an AFO remains limited because of a lack of

118 sufficient information regarding the effects of different kinds of AFOs on gait. Considering the effect

119 of different kinds of AFOs on gait, the stiffness of an AFO is considered a key determinant of its

120 functional effect. In this context, stiffness is defined as the slope of the curve of resistance (resistive

121 moment) versus ankle joint angle when an AFO is deformed in plantar flexion or dorsiflexion $[3,4]$.

122 A systematic review showed that AFO stiffness mainly affected ankle kinematics, suggesting that greater stiffness could generally result in a decreased peak ankle joint angle in both plantar flexion and dorsiflexion [5]. Some AFOs have been developed to assist insufficient muscle activity through resistance [6,7]. Therefore, it is necessary to know the effect of different kinds of AFOs with different moments of resistance on the gait of patients.

Based on the results of gait analysis of patients with stroke, an AFO with an oil damper (AFOOD) was developed to assist the dorsiflexors in the loading response $[8,9]$. The resistive moment is generated by an oil damper when the ankle joint moves to plantar flexion and the ankle joint of the

130 AFO-OD moves freely to dorsiflexion. Gait improvement with AFO-ODs has been shown in some previous studies, but the results were reported in comparison to gait without an AFO [10,11]. A randomized controlled trial comparing AFO-ODs and AFOs with plantar flexion stops in 40 patients in the subacute phase of stroke found that the function of AFOs in plantar flexion affected the

134 forward inclination of the pelvis and thorax during gait [12]. As the next step, AFO function during dorsiflexion should be compared. 

impedes smooth movement of the ankle during the stance phase $[1,15,16]$. The dorsiflexion angle in mid-stance affects the progression of the center of pressure, affecting the ankle joint moment and power [17]. However, to our knowledge, no studies have compared the ankle joint kinetics between

141 AFOs with and without dorsiflexion resistance and how these differences affect the gait of patients

142 with stroke. In the present randomized controlled trial, the gait with an AFO-OD, which allowed the 143 ankle joint to move freely with no resistance, was compared to the gait with a nonarticulated AFO, 144 which had dorsiflexion resistance. Both AFOs offered plantarflexion resistance. The hypothesis was that the angle of dorsiflexion in mid-stance was restricted when walking with a nonarticulated AFO and that this restriction affected the ankle joint kinetics in stance.

\section{Methods}

2.1 Participants

The participants were patients with subacute stroke (more than 14 and less than 180 days after onset). Patients in the subacute phase were selected for this study because the gait of patients in the chronic phase is affected largely by AFOs in daily use. The participants were inpatients at a rehabilitation center who were walking independently with or without assistive devices and had been prescribed gait training with an AFO. The following exclusion criteria were applied: pre-existing pathology affecting the central nervous system or neuromuscular system and communication problems. Participants whose step length had a negative value were excluded from the analysis because a negative step length indicates an active reduction in paretic propulsion due to severe ankle plantar flexor impairment resulted in a different gait pattern [18]. All participants underwent gait training under physiotherapists' supervision. None had previously used an AFO, and all were enrolled in the 

in stance phase as the main outcome, assuming a variance of $5.3^{\circ}$ and a difference of $5^{\circ}$ between the articulated and nonarticulated AFO groups [19]. The significance level was 0.05, and the power was 0.8. Using the G-Power program (Kiel University, Kiel, Germany), the required sample size per group was calculated as 15 . To account for data loss, 41 participants were recruited for this study. The study protocol was approved by the ethics committees of the International University of Health \& Welfare and Nakaizu Rehabilitation Center. Written informed consent was obtained from all participants prior to their participation in this study.

\subsection{Experimental AFO} and a nonarticulated AFO. The AFO-OD had a mechanical ankle joint with an oil damper (Figure 1) [9] that generated a resistive moment when the ankle joint moved in plantar flexion. The resistive moment could be changed by rotating a screw at the top of the oil damper from 1 (flexible) to 4 (rigid). In the present study, the screw was set to 3 because this level is used most frequently by patients in the subacute phase of stroke [12]. The AFO-OD allowed the ankle joint to move freely in dorsiflexion without any resistance. The nonarticulated AFO used in this study had no mechanical joint and an ankle trim line behind the malleoli (Figure 1). The initial ankle joint angle was set at neutral when the patient wore an AFO in both groups. Given that the AFOs were not custom-made, we prepared 6

179 AFOs for each group, namely, right and left AFOs in each of 3 sizes (small, medium, and large). The resistive moment of all AFOs was measured using a goniometer, a force meter (digital force gauge, IMADA, Japan), and a metronome. Because of differences in the inherent characteristics of plastic, which has elasticity, and the oil damper, which has viscosity, the measurement was static for the nonarticulated AFO and dynamic for the AFO-OD. Table 1 shows the characteristics of the AFOs as 

or at fast speed (approximately $20 \mathrm{deg} / \mathrm{s}$ ). System, Ltd., Oxford, UK) and 6 AMTI force plates (Advanced Mechanical Technology, Inc., Phoenix, AZ). Reflective markers were attached to the participants' bodies in accordance with the Plug-In Gait model. Additional markers were used on the medial side of the knee and ankle joints to precisely calculate the joint centers. Marker trajectories and force plate data were captured at a sampling frequency of $100 \mathrm{~Hz}$. The participants wore shoes (V-step; Pacific Supply, Osaka, Japan) that were suitable size for each condition with and without an AFO. The use of a cane was permitted, but it had to be used in a consistent manner when walking without an AFO and with either type of AFO.

First, shod gait with no AFO was measured at each participant's self-selected walking speed nonarticulated AFO group. Then, the participants started gait training sessions for $1 \mathrm{~h}$ daily over 2 weeks under the supervision of physiotherapists. They also participated in general physiotherapy, including range of motion ( $\mathrm{ROM})$ exercises, balance training, and muscle training. There were no differences in the physiotherapy processes between the two AFO groups. After 2 weeks of training, gait with the allocated AFOs was measured using the same procedure. The participants did not use any kind of AFOs in their daily lives except during gait training.

Marker trajectories and force plate data were low-pass filtered by a second-order Butterworth filter with cutoff frequencies of 6 and $18 \mathrm{~Hz}$, respectively. The gait cycle time and duration of each gait phase were defined using force plate data. The trajectories of heel markers were used if the participant walked with a cane on their nonparetic side. Given that participants were in subacute stroke, 
loading response, single-stance, preswing, and swing phases of the paretic limb. The measured spatial

210 and temporal parameters were velocity, step length, and duration of each gait cycle. The anterior and

211 posterior components of the ground reaction force were also calculated. The angle of inclination of 212 the shank segment relative to the vertical (shank-to-vertical angle, SVA) in the sagittal plane was 213 calculated in view of its importance when evaluating gait with an AFO [20]. Ankle joint kinematics 214 and kinetics were calculated using an inverse dynamic model. Step length was normalized by each 215 participant's body height, and ground reaction force, joint moment, and power were normalized by the 216 participant's body weight. Kinematic and kinetic data were obtained for the paretic limb. All calculations were performed using Visual 3D software version 6 (C-Motion Inc., Kingston, ON, 218 Canada).

2.4 Statistical analysis

All gait parameters are presented as the average of at least three gait cycles for each condition (without an AFO before training and with an AFO after training). The data were assessed for normality by the Shapiro-Wilk test. For the baseline data, differences between the two AFO groups in all gait parameters without AFOs were compared using the unpaired $\mathrm{t}$ test or the Mann-Whitney $U$ test. For the comparison of gain between the with- and without-AFO conditions, two-way mixed ANOVA was performed for normally distributed data, with wearing or not wearing an AFO as a paired factor and type of AFO as a nonpaired factor. If no interaction was found, the main effects of the two factors were evaluated. If an interaction was found, the effect of wearing or not wearing an AFO was compared within each group using one-way ANOVA. For data that were not normally distributed, the effect of wearing or not wearing an AFO was compared using the Wilcoxon signed-rank test, and the effect of the type of AFO worn was examined using the Mann-Whitney $U$ test. The correlation coefficient was also calculated between parameters that showed significant interactions in each group. A p-value $<$ 


\section{Results}

One participant in each group was withdrawn because of missing data during the study period.

Three participants were excluded from the analysis due to negative paretic-to-nonparetic step length at baseline, leaving data from 36 participants for inclusion in the analysis (AFO-OD group, $\mathrm{n}=17$; nonarticulated AFO group, $\mathrm{n}=19$ ), as shown in Figure 2. There was no significant difference between the groups in age, body height and weight or days since stroke onset at baseline (Table 2). Eleven of 17 participants in the AFO-OD group and 15 of 19 in the nonarticulated AFO group used a cane.

In the baseline comparison, no difference was found in any gait parameter. No significant main effects of AFO group were found for any of the parameters according to the type of AFO worn $(\mathrm{p}>0.05)$ (data not shown in the tables). The results for temporal and spatial factors, ground reaction forces, and SVA angle are shown in Table 3. Significant interactions were found for the range of SVA during the single-stance phase $(\mathrm{p}=0.029, \mathrm{~F}=5.14)$. The range of SVA during single stance was significantly increased in the AFO-OD group $(\mathrm{p}=0.006, \mathrm{~F}=10.14)$, but not in the nonarticulated AFO group. A significant main effect of AFO use was found according to whether an AFO was worn in both groups for many parameters, including velocity, paretic to nonparetic step length ( $p=0.01, F=12.73$ ), nonparetic to paretic step length $(\mathrm{p}<0.001, \mathrm{~F}=20.19)$, single-stance time $(\mathrm{p}=0.003, \mathrm{~F}=9.97)$, anterior component of the ground reaction force $(p=0.006, F=8.73)$, SVA at paretic initial contact $(\mathrm{p}=0.024, \mathrm{~F}=5.55)$, and SVA at nonparetic initial contact $(\mathrm{p}<0.001, \mathrm{~F}=18.35)$, all of which were significantly increased with AFO use. Temporal factors, cycle time $(\mathrm{p}<0.001, \mathrm{~F}=30.12)$, loading response time and preswing time were significantly decreased by the use of an AFO. 
OD group ( $p=0.001, F=16.76)$ but not in the nonarticulated AFO group. As a significant main effect of AFO use, the peak dorsiflexion ankle joint angle was increased $(\mathrm{p}=0.024, \mathrm{~F}=5.58)$, and the peak plantar flexion moment in stance was increased $(\mathrm{p}<0.001, \mathrm{~F}=24.32)$ in both groups when an AFO was worn. The p-values of each AFO group for parameters that were not normally distributed are shown in Tables 3 and 4. Figure 3 shows the ankle power graph in paretic stance. The bold lines show the average of the participants in each AFO group, and the thin lines show the standard deviation. In the AFO-OD group, the negative power was increased when the participants walked with the AFO-OD, but there were no changes in the nonarticulated AFO group.

Figure 4 shows the relationship of the range of SVA in single stance with peak power absorption during gait for each AFO type. Each mark represents an individual participant (white, AFO-OD group; black, nonarticulated AFO group). The SVA range was negatively correlated with peak power absorption in the nonarticulated AFO group $(\mathrm{r}=-0.81, \mathrm{p}<0.001)$. However, these parameters were restricted to a narrow range in this AFO group, with the exception of two participants, both of whom had a large SVA range of more than $15^{\circ}$, even when walking without an AFO. The ranges of SVA during the single-stance and peak power absorption phases were variable, and the correlation coefficient was not significant $(\mathrm{r}=-0.47)$ in the AFO-OD group.

\section{Discussion}

In this study, the effect of two kinds of AFOs on the gait of patients with stroke was investigated. The mechanical characteristics of both types of AFOs used in this study were compared. The plantar flexion resistance was 3.12 to $3.19 \mathrm{Nm} / \mathrm{deg}$ in the nonarticulated AFO group. The ankle joint angle at initial contact was $-2.5 \mathrm{deg}$, and the maximum plantar flexion was $-6.5 \mathrm{deg}$, as shown in Table 3 . This means that the ankle joint moved to plantar flexion at approximately 4 deg; thus, the peak plantar 
the AFO-OD was 3.08 to $5.81 \mathrm{Nm}$ These results indicate that the peak plantar flexion resistance of the AFO-OD was smaller than that of the nonarticulated AFO. Plantar flexion resistance can affect the gait during the loading response [21]. However, no difference was found in gait parameters in the loading phase in this study. Dorsiflexion resistance was 1.75 to $1.92 \mathrm{Nm} / \mathrm{deg}$ in the nonarticulated AFO. In a systematic review that investigated the impact of AFO stiffness on gait, the reported range of stiffness in the included studies was $0.02-8.17 \mathrm{Nm} / \mathrm{deg}$ [5]. Another study comparing 4 kinds of AFOs showed a stiffness range of 0.2 to $2.0 \mathrm{Nm} / \mathrm{deg}$ [22]. Referring to these results, the dorsiflexion stiffness of the nonarticulated AFO used in this study was moderate to relatively stiff.

In our hypothesis, we predicted that the peak ankle joint angle in dorsiflexion would be larger when the AFO-OD was used and would affect ankle kinetics. Our results showed that the peak dorsiflexion angle with an AFO was $6.73^{\circ}$ in the AFO-OD group and $1.39^{\circ}$ in the nonarticulated AFO group, with no significant interaction $(\mathrm{p}=0.062)$. However, the range in SVA during the single-stance phase was increased in the AFO-OD group, and no difference was found in the nonarticulated AFO group. The range in SVA was related to the paretic-to-nonparetic step length. In this study, no interaction was found for paretic-to-nonparetic step length $(\mathrm{p}=0.065)$. Regarding ankle joint kinetics, the peak ankle power absorption was increased in the AFO-OD group, but no difference was found in the nonarticulated AFO group. Therefore, the hypothesis was partially proven.

Ankle power absorption is related to the eccentric contraction of the plantar flexors in mid stance [17]. It is obtained by multiplying the plantar flexion moment by the angular velocity of ankle joint dorsiflexion. Based on the results of the present study, the plantar flexion moment was increased in both AFO groups. Therefore, the difference of the power between AFO groups was probably caused by the difference in the angular velocity of ankle joint dorsiflexion, which in turn was related to the increased range in SVA in the AFO-OD group. In previous studies, the dorsiflexion resistance of AFOs was thought to be important to compensate for the insufficient activity of the plantar flexors during 
gait $[13,14,23]$. However, the results of this study showed that the moderate to relatively stiff dorsiflexion resistance of an AFO can impede ankle kinetics in paretic stance. As shown in Figure 4,

307 the peak power absorption was closely related to the range in SVA in the nonarticulated AFO group due to assisted dorsiflexion by the AFO. However, it was restricted within a narrow range. On the other hand, the peak power absorption was variable in the AFO-OD group because it depended on the plantar flexor activity of each participant. Although dorsiflexion was not supported by the AFO, no participant in the AFO-OD group collapsed during paretic stance.

The effect of plantar flexion resistance of AFOs has been shown in previous studies [1,2], but to the best of our knowledge, the effect of dorsiflexion resistance is still unclear. To determine the effect of AFO function in dorsiflexion, forward rotation of the shank in paretic stance is a key parameter $[20,24]$. When the patients walked wearing the AFO with plantar flexion stops, the ankle joint was dorsiflexed, and the knee joint was flexed, thereby excessively pushing the shank forward in loading response $[25,26]$. In this case, it is necessary for the AFO to assist the plantar flexors in preventing excessive forward rotation of the shank. The AFO-OD allows plantar flexion movement with resistance during the loading response, and the shank is not pushed excessively forward $[10,26]$. This extended plantar flexors store energy and generate propulsive force in late stance [27, 28]. However, there was no significant increase in ankle power generation in either AFO group. The interaction between groups was not significant $(\mathrm{p}=0.055$ ). Both types of AFO used in our study resisted plantar 

The AFO-OD was not able to increase the power generation in late stance, even though the power absorption was increased in stance phase. to the results of previous systematic reviews related to gait improvement with AFOs $[1,2]$. Because the participants in the present study were patients in the subacute phase, the results included the effects of not only AFO use but also gait recovery and physiotherapy. Improved gait parameters after 2 weeks were similar to the results shown in a systematic review of gait improvement by exercise-based rehabilitation [30]. However, the effects of the use of AFO, gait recovery, and physiotherapy could not be differentiated in this study.

This study had some other limitations. First, the plantar flexion resistive moments of the AFOOD and nonarticulated AFO were the same for each study participant. The results of the present study might have been different if the resistive moment of the AFOs had been adjusted for each individual participant. Second, there was a difference in the resistance to plantar flexion between the two types of AFO used in this study in terms of viscosity and elasticity. Most participants in both AFO groups used canes on their nonparetic side. The use of canes indirectly affected the ankle power of the paretic stance, but these participants could not walk without canes. Moreover, this study was conducted at a rehabilitation hospital, and thus, further study in different settings will be necessary to explore the generalizability of the results.

\section{Conclusion}

This randomized controlled trial assessed the effect of a nonarticulated AFO, which resists dorsiflexion, with those of an AFO-OD, which does not. Both AFOs had plantar flexion resistance. 
stance phase and peak power absorption were significantly increased in the AFO-OD group, but no difference was found in the nonarticulated AFO group. These gait parameters were correlated with one another in the nonarticulated AFO group but were restricted to a narrow range in most patients. Power generation was not increased in either AFO group. The results of this study showed that AFO with plantar flexion resistance but without dorsiflexion resistance improved ankle power absorption, but not power generation, in a paretic stance. (3,621 words)

\section{Declarations}

- Ethics approval and consent to participate

The study protocol was approved by the ethics committees of the International University of Health

\& Welfare and Nakaizu Rehabilitation Center. Written informed consent was obtained from all participants prior to their participation in this study.

All authors give the permission of the publication of the article to the publisher.

Raw data were generated at Nakaizu Rehabilitation Hospital. Derived data supporting the findings

369 of this study are available from the corresponding author S.Y. on request.

$370 \quad \cdot$ Competing interests

371 The authors are unaware of any conflicts of interest concerning this study.

$372 \cdot$ Funding

373 This study was supported by JSPS KAKENHI grant number 17K01577.

374 - Authors' contributions

375 S.Y., N.M., Y.K., and Y.O. designed the study. N.M. and Y.K. recruited patients and 376 collected the data. S.Y., S.T., and A.D. analyzed the data. S.Y. drafted the manuscript, 377 and A.D. revised the final version. 
379 The authors thank all patients who participated in this study and the physiotherapists who assisted with 380 gait training and obtaining measurements.

381

382

383

384

\section{References}

385

1. Daryabor A, Arazpour M, Aminian G: Effect of different designs of ankle-foot orthoses on gait in patients with stroke: A systematic review. Gait \& Posture 2018, 62: 268-279.

2. Tyson SF, Sadeghi-Demneh E, Nester CL: A systematic review and meta-analysis of the effect of an ankle-foot orthosis on gait biomechanics after stroke. Clin Rehabil 2013, 27: 879-891.

3. Major RE, Hewart PJ, MacDonald AM: A new structural concept in molded fixed ankle foot orthoses and comparison of the bending stiffness of four constructions. Prosthet. Orthot. Int.2004, 28(1): 44-48.

4. Bregman DJJ, Rozumalski A< Koops D, Groot V, Schwartz M, Harlaar J: A new method for evaluating ankle foot orthosis characteristics: BRUCE, Gait Posture 2009, 30(2):144-149.

5. Totah D, Menon M, Jones-Hershinow C, Barton K, Gates DH: The impact of ankle-foot orthosis stiffness on gait: A systematic literature review. Gait \& Posture 2019, 69: 101-111.

6. Arch ES, Stanhope SJ: Passive-dynamic ankle-foot orthoses substitute for ankle strength while causing adaptive gait strategies: a feasibility study, Annals of Biomedical Engineering 2015, 43(2): 442-450.

7. Waterval NFJ, Brehm MA, Altmann VC, Koopman FS, Den Boer JJ, Harlar, J, Nollet F:

400 Stiffness-optimized ankle-foot orthoses improve walking energy cost compared to conventional 401 orthoses in neuromuscular disorders: a prospective uncontrolled intervention study, IEEE Trans Neural Syst Rehabil. Eng. 2020, 28(10):2296-2304. 

ankle-foot orthoses on hemiplegic gait, Gait \& Posture 1993, 1:27-34.

9. Yamamoto S, Hagiwara A, MizobeT, Yokoyama O, Yasui T:Development of an ankle-foot orthosis with an oil damper. Prosthet Orthot Int. 2005, 29: 209-219.

10. Yamamoto S, Fuchi M, Yasui T: Change of rocker function in the gait of stroke patients using an ankle foot orthosis with an oil damper: immediate changes and the short-term effects, Prosthet Orthot Int. 2011, 35(4): 350-359.

11. Yamamoto S, Tomokiyo N, Yasui T, Kawaguchi T: Effects of plantar flexion resistive moment generated by an ankle-foot orthosis with an oil damper on the gait of stroke patients: a pilot study. Prosthet Orthot Int 2013, 37(3): 212-221.

12. Yamamoto S, Tanaka S, Motojima N: Comparison of ankle-foot orthoses with plantar flexion stop and plantar flexion resistance in the gait of stroke patients: A randomized controlled trial. Prosthet. Orthot. Int. 2018, 42(5): 544-553.

13.Harper NG, Russel Esposito E, Wilken JM, Neptune RR: The influence of ankle-foot orthosis stiffness on walking performance in individuals with lower-limb impairment, Clin. Biomech. (Bristol, Avon) 2014. 29:877-884.

14. Malas BS: What reliables influence the ability of an AFO to improve function and when are they

15. Alam M, Choudhury IA, Mamat AB:Mechanism and design analysis of articulated ankle foot indicated? Clin. Orthop. Relat. Res 2011, 469:1308-1314. of an articulated ankle-foot orthosis with resistance-adjustable joints on lower limb joint kinematics and kinetics during gait in individuals post-stroke. Clin. Biomech.2018, 59: 47-55. 
18. Balasubramanian CK, Bowden MG, Neptune RR, Kautz SA: Relationship between step length asymmetry and walking performance in subjects with chronic hemiparesis. Arch Phys Med Rehabil. 2007, 88(1): 43-49.

19. Gok H, Kucukdeveci A, Altinkaynak H, Yavuzer G, Ergin S: Effects of ankle-foot orthoses on hemiplaretic gait. Clin Rehabil 2003, 17: 137-139.

20. Kerkum YL, Houdijk H, Brehm MA, Buizer A, Kessels MLS, Sterk A, van den Noort JC, Harlaar J: The Shank-to Vertical Angle as a parameter to evaluate tuing of Ankle-Foot Orthoses. Gait \& Posture 2014, 42:269-274.

21. Nolan KJ, Yarossi M: Preservation of the first rocker is related to increases in gait speed in individuals with hemiplegia and AFO, Clin. Biomech. (Bristol, Avon) 2010, 26: 655-660.

22.Waterval NFJ, Breham MA, Harlaar J, Nollet F, Description of orthotic properties and effect evaluation of ankle foot orthoses in non-spastic calf muscle weakness, J. Rehabil. Med. 2020, 52: jrm00026.

23. Harlaar J, Brehm M, Becher JG, Bregmen J, Buurke J, Holtkamp F, DeGroot V, Nollet F: Studies examining the efficacy of ankle foot orthoses should report activity level and mechanical evidence, Prosthet. Orthot. Int. 2010, 34:327-335

24. Owen E, The importance of bein earnest about shank and thigh kinematics expecialy when usin ankle-foot-orthoses, Prosthet. Orthot. Int. 2010, 34:254-269.

25. Mulroy SJ, Eberly VJ, Gronely JK, Walter W, Newsam CJ: Effect of AFO design on walking after stroke: Impact of ankle plantar flexion contracture. Prosthet Orthot Int. 2010, 24:277-292.

26. Ohata K, Yasui T, Tsuboyama T, Ichihashi N: Effects of an ankle-foot orthosis with oil damper on muscle activity in adults after stroke. Gait \& Posture 2011, 33:102-107.

27. Neptune RR, Kautz SA, Zajac FE: Contributions of the individual ankle plantar flexors to suppout, forward progression and swing initiation during walking, J. Biomech. 2001, 34:1387-1398. 
451

452

453

454

455

456

457

458

459

460

461

462

463

464

465

466

467

468

469

470

471

472

473

474

475

476

28. Fickey SN, Browne MG, Franz JR: Biomechanical effects of augmented ankle power output during human walking, J. Experimental Biology 2018, 221:Jeb1821113, doi:10.1242.

29.Desloovere K, Molenaers G, Van Gestel L, Huenaerts C, Van Campenhout A, Callewaert B, Vande Walle P, Seyler J, How can push-off be preserved during use of an ankle foot orthosis in children with hemiplegia? A prospective controlled study, Gait \& Posture 2006, 24:142-151.

30. An M, Shaughnessy M: The effects of exercise-based rehabilitation on balance and gait for stroke patients: a systematic review, J. Neurosci Nurs 2011, 43(6):298-307.

\section{Figure captions}

Fig. 1. The ankle-foot orthoses used in this study. Left: An ankle-foot orthosis with an oil damper (AFO-OD). Right: A nonarticulated ankle-foot orthosis.

Fig. 2 Consolidated Standard of Reporting Trials (CONSORT) flowchart.

Fig. 3 Graph of average ankle power in paretic stance in each AFO group ( $n=17$ in the AFO-OD group, $\mathrm{n}=19$ in the nonarticulated group)

bold line, average; thin line, standard deviation

Fig. 4 Relationship between shank-to-vertical angle range and ankle peak power absorption.

SVA, shank-to-vertical angle; AFO-OD, ankle-foot orthosis with oil damper; nonarticulated AFO, nonarticulated ankle-foot orthosis

Table 1 Characteristics of the AFOs used in this study

Table 2 Patient characteristics

Table 3 Result of temporal and spatial factors, ground reaction forces, and shank-to-vertical angle

Table 4 Result of ankle joint kinematics and kinetics 


\begin{tabular}{lllllll}
\hline & AFO-OD & & \multicolumn{4}{c}{ Non-articulated AFO } \\
& Small & Medium & Large & Small & Medium & Large \\
\hline Weight (g) & 395 & 430 & 500 & 310 & 360 & 385 \\
Calf height (cm) & 32.0 & 35.0 & 37.0 & 32.0 & 35.0 & 37.0 \\
Foot length (cm) & 22.5 & 24.5 & 27.0 & 22.5 & 24.5 & 27.0 \\
Width (cm) & N.A. & N.A. & N.A. & 6.7 & 6.7 & 7.0 \\
$\begin{array}{l}\text { Wall thickness } \\
\text { (cm) }\end{array}$ & 0.3 & 0.3 & 0.3 & 0.3 & 0.3 & 0.3 \\
$\begin{array}{l}\text { Plantar flexion } \\
\text { resistance }\end{array}$ & $3.12 \mathrm{Nm}^{*}$ & $3.38 \mathrm{Nm}^{*}$ & $3.08 \mathrm{Nm}^{*}$ & 3.19 & 3.16 & 3.12 \\
$\begin{array}{l}\text { Dorsi flexion } \\
\text { resistance }\end{array}$ & N.46 Nm** & $5.81 \mathrm{Nm}^{* *}$ & $5.74 \mathrm{Nm} * *$ & $\mathrm{Nm} / \mathrm{deg}$ & $\mathrm{Nm} / \mathrm{deg}$ & $\mathrm{Nm} / \mathrm{deg}$ \\
\hline AFO-OD: & N.A. & N.A. & 1.80 & 1.75 & 1.92 \\
& & & & $\mathrm{Nm} / \mathrm{deg}$ & $\mathrm{Nm} / \mathrm{deg}$ & $\mathrm{Nm} / \mathrm{deg}$ \\
\hline
\end{tabular}

AFO-OD: ankle-foot orthosis with an oil damper; NA-AFO: non-articulated AFO 


\begin{tabular}{llll}
\hline & $\begin{array}{l}\text { AFO-OD } \\
(\mathbf{n}=\mathbf{1 7})\end{array}$ & $\begin{array}{l}\text { Nonarticulated AFO } \\
(\mathbf{n}=\mathbf{1 9})\end{array}$ \\
\hline Sex & Male: 13, Female:4 & Male: 15, Female: 4 & \\
Age, years & $56.2(12.9)$ & $60.5(9.7)$ & $\mathrm{ns}$ \\
Body height, cm & $167.2(7.6)$ & $165.5(7.6)$ & $\mathrm{ns}$ \\
Body weight, $\mathbf{k g}$ & $58.7(9.5)$ & $64.5(11.6)$ & $\mathrm{ns}$ \\
Diagnosis & Cerebral hemorrhage: 10, & Cerebral hemorrhage: 7, & \\
Paretic side & Cerebral infarction : 7 & Cerebral infarction : 12 & \\
$\begin{array}{l}\text { Days since onset } \\
\text { Brunnstrom stage }\end{array}$ & $\begin{array}{l}\text { Right: } 8, \text { Left: } 9 \\
\text { of lower extremities }\end{array}$ & II: 2, III: 7, IV: $3, \mathrm{~V}: 5$ & Right: 11, Left: 8 \\
Manual ROM test & & $65.8(39.5)$, Min: 22, Max: 147 & ns \\
of ankle joint & $0^{\circ}: 1,5^{\circ}: 8,10^{\circ}: 5,15^{\circ}: 3$ & $-5^{\circ}: 1,0^{\circ}: 2,5^{\circ}: 4,10^{\circ}: 6,15^{\circ}:$ & \\
Modified Ashworth & & $5,20^{\circ}: 0,25^{\circ}: 1$ & \\
Scale & $0: 5,1: 3,1+: 4,2: 5$ & $0: 7,1: 4,1+: 6,2: 2$ & \\
Use of cane & Yes: 11, No: 6 & Yes: 15, No: 4 & \\
\hline
\end{tabular}

502

503

504

505

506

507

508

509

510

511

512

513

514

515

516

517

518

519

Mean (standard deviation)

AFO-OD: ankle-foot orthosis with an oil damper; NA-AFO: non-articulated AFO; ns: not significant; ROM: range of motion 
Table 3 Results of temporal and spatial factors, ground reaction forces, and shank-to-vertical angle

\begin{tabular}{|c|c|c|c|c|c|c|c|c|}
\hline & \multicolumn{2}{|l|}{ AFO-OD } & \multicolumn{2}{|c|}{ Nonarticulated AFO } & \multirow[t]{2}{*}{ Interaction } & \multirow{2}{*}{$\begin{array}{l}\text { Main effect of } \\
\text { AFO use } \\
\text { With vs } \\
\text { without }\end{array}$} & \multicolumn{2}{|c|}{ With vs without } \\
\hline & Without & With & Without & With & & & AFO-OD & $\begin{array}{l}\text { Nonarticulated } \\
\text { AFO }\end{array}$ \\
\hline \multicolumn{9}{|l|}{ Spatial and temporal } \\
\hline Velocity $(\mathbf{m} / \mathbf{s})^{\#}$ & $\begin{array}{l}0.180 \\
(0.136)\end{array}$ & $\begin{array}{l}0.332 \\
(0.189)\end{array}$ & $\begin{array}{l}0.222 \\
(0.174)\end{array}$ & $\begin{array}{l}0.367 \\
(0.120)\end{array}$ & & & $<0.001^{* *}$ & $0.001^{* *}$ \\
\hline $\begin{array}{l}\text { Step length (paretic to non- } \\
\text { paretic)/height }\end{array}$ & $\begin{array}{l}0.145 \\
(0.067)\end{array}$ & $\begin{array}{l}0.200 \\
(0.083)\end{array}$ & $\begin{array}{l}0.171 \\
(0.068)\end{array}$ & $\begin{array}{l}0.189 \\
(0.059)\end{array}$ & 0.065 & $0.01 *$ & & \\
\hline $\begin{array}{l}\text { Step length (non-paretic to } \\
\text { paretic)/height }\end{array}$ & $\begin{array}{l}0.168 \\
(0.082)\end{array}$ & $\begin{array}{l}0.209 \\
(0.073)\end{array}$ & $\begin{array}{l}0.159 \\
(0.074)\end{array}$ & $\begin{array}{l}0.202 \\
(0.061)\end{array}$ & 0.921 & $<0.001^{* *}$ & & \\
\hline Cycle time (s) & $\begin{array}{l}2.238 \\
(0.549)\end{array}$ & $\begin{array}{l}1.886 \\
(0.623)\end{array}$ & $\begin{array}{l}2.258 \\
(0.440)\end{array}$ & $\begin{array}{l}1.854 \\
(0.563)\end{array}$ & 0.827 & $<0.001 * *$ & & \\
\hline Loading response time (s) & $\begin{array}{l}0.400 \\
(0.218)\end{array}$ & $\begin{array}{l}0.308 \\
(0.242)\end{array}$ & $\begin{array}{l}0.425 \\
(0.168)\end{array}$ & $\begin{array}{l}0.317 \\
(0.129)\end{array}$ & & & $0.011^{* *}$ & $0.005^{* *}$ \\
\hline Single stance time (s) & $\begin{array}{l}0.376 \\
(0.112)\end{array}$ & $\begin{array}{l}0.428 \\
(0.104)\end{array}$ & $\begin{array}{l}0.400 \\
(0.101)\end{array}$ & $\begin{array}{l}0.429 \\
(0.118)\end{array}$ & 0.379 & $0.003^{* *}$ & & \\
\hline Preswing time $(\mathbf{s})^{\#}$ & & & & & & & $<0.001 * *$ & $0.001^{* *}$ \\
\hline
\end{tabular}




\begin{tabular}{|c|c|c|c|c|c|c|c|c|}
\hline & $\begin{array}{l}0.858 \\
(0.604)\end{array}$ & $\begin{array}{l}0.425 \\
(0.525)\end{array}$ & $\begin{array}{l}0.717 \\
(0.396)\end{array}$ & $\begin{array}{l}0.386 \\
(0.360)\end{array}$ & & & & \\
\hline Swing time (s) ${ }^{\#}$ & $\begin{array}{l}0.629 \\
(0.182)\end{array}$ & $\begin{array}{l}0.593 \\
(0.152)\end{array}$ & $\begin{array}{l}0.669 \\
(0.141)\end{array}$ & $\begin{array}{l}0.583 \\
(0.117)\end{array}$ & & & $0.031^{*}$ & $0.013 *$ \\
\hline \multicolumn{9}{|l|}{ Ground reaction force } \\
\hline Peak posterior (N/kg) & $\begin{array}{l}-0.815 \\
(0.384)\end{array}$ & $\begin{array}{l}-0.904 \\
(0.462)\end{array}$ & $\begin{array}{l}-0.814 \\
(0.193)\end{array}$ & $\begin{array}{l}-0.857 \\
(0.207)\end{array}$ & 0.399 & 0.125 & & \\
\hline Peak anterior $(\mathrm{N} / \mathrm{kg})$ & $\begin{array}{l}0.368 \\
(0.163)\end{array}$ & $\begin{array}{l}0.449 \\
(0.246)\end{array}$ & $\begin{array}{l}0.313 \\
(0.210)\end{array}$ & $\begin{array}{l}0.495 \\
(0.305)\end{array}$ & 0.273 & $0.006^{* *}$ & & \\
\hline \multicolumn{9}{|l|}{ Shank to vertical angle } \\
\hline Initial contact $\left({ }^{\circ}\right)$ & $\begin{array}{l}-5.276 \\
(4.357)\end{array}$ & $\begin{array}{l}-2.793 \\
(5.089)\end{array}$ & $\begin{array}{l}-6.828 \\
(6.117)\end{array}$ & $\begin{array}{l}-5.772 \\
(5.397)\end{array}$ & 0.339 & $0.024^{*}$ & & \\
\hline $\begin{array}{l}\text { Initial contact of non- } \\
\text { paretic limb }\left({ }^{\circ}\right)\end{array}$ & $\begin{array}{l}8.422 \\
(7.818)\end{array}$ & $\begin{array}{l}14.209 \\
(6.330)\end{array}$ & $\begin{array}{l}7.615 \\
(4.389)\end{array}$ & $\begin{array}{l}10.376 \\
(4.992)\end{array}$ & 0.124 & $<0.001^{* *}$ & & \\
\hline Range in single stance $\left({ }^{\circ}\right)$ & $\begin{array}{l}4.774 \\
(3.499)\end{array}$ & $\begin{array}{l}8.088 \\
(4.623)\end{array}$ & $\begin{array}{l}5.364 \\
(4.034)\end{array}$ & $\begin{array}{l}5.642 \\
(4.888)\end{array}$ & $0.029 *$ & & $0.006^{* *}$ & 0.614 \\
\hline
\end{tabular}

AFO-OD: AFO with an oil damper; shank to vertical angle: forward inclination +; mean (standard deviation) for normally distributed data; median (interquatile range) for non-normally distributed data; ns: not significant

${ }^{*} \mathrm{p}<0.05 ; * * \mathrm{p}<0.01 ;{ }^{*}$ Not normally distributed 
Table 4 Result of ankle joint kinematics and kinetics

\begin{tabular}{llllll} 
AFO-OD & \multicolumn{2}{l}{$\begin{array}{l}\text { Nonarticulated } \\
\text { AFO }\end{array}$} & Interaction & $\begin{array}{l}\text { Main } \\
\text { effect of } \\
\text { AFO use }\end{array}$ \\
Without & With Without With & $\begin{array}{l}\text { With vs } \\
\text { without }\end{array}$ & $\begin{array}{l}\text { AFO- } \\
\text { OD }\end{array}$ & $\begin{array}{l}\text { Nonarticulated } \\
\text { AFO }\end{array}$
\end{tabular}

\begin{tabular}{|c|c|c|c|c|c|c|c|c|}
\hline & (1) & 然 & (1) & & & without & OD & AFO \\
\hline \multicolumn{9}{|l|}{ Ankle angle } \\
\hline Initial contact $\left({ }^{\circ}\right)^{\#}$ & $\begin{array}{l}-6.73 \\
(3.97)\end{array}$ & $\begin{array}{l}1.98 \\
(5.75)\end{array}$ & $\begin{array}{l}-10.37 \\
(9.92)\end{array}$ & $\begin{array}{l}-2.48 \\
(4.32)\end{array}$ & & & $0.001 * *$ & $0.001 * *$ \\
\hline $\begin{array}{l}\text { Peak plantar flexion in loading } \\
\text { response }\left({ }^{\circ}\right)^{\#}\end{array}$ & $\begin{array}{l}-9.73 \\
(5.44)\end{array}$ & $\begin{array}{l}-1.25 \\
(6.15)\end{array}$ & $\begin{array}{l}-10.81 \\
(8.38)\end{array}$ & $\begin{array}{l}-6.54 \\
(4.26)\end{array}$ & & & $0.003 * *$ & $<0.001 * *$ \\
\hline $\begin{array}{l}\text { Peak dorsiflexion in single stance } \\
\left({ }^{\circ}\right)\end{array}$ & $\begin{array}{l}2.60 \\
(6.91)\end{array}$ & $\begin{array}{l}6.73 \\
(6.54)\end{array}$ & $\begin{array}{l}0.97 \\
(4.85)\end{array}$ & $\begin{array}{l}1.39 \\
(4.76)\end{array}$ & 0.062 & $0.024 *$ & & \\
\hline $\begin{array}{l}\text { Peak plantar flexion in pre-swing } \\
\left({ }^{\circ}\right)^{\#}\end{array}$ & $\begin{array}{l}-3.14 \\
(4.65)\end{array}$ & $\begin{array}{l}3.06 \\
(7.19)\end{array}$ & $\begin{array}{l}-5.08 \\
(8.53)\end{array}$ & $\begin{array}{l}-1.07 \\
(3.78)\end{array}$ & & & $0.003 * *$ & $0.008 * *$ \\
\hline Peak dorsiflexion in swing $\left({ }^{\circ}\right)^{\#}$ & $\begin{array}{l}-0.89 \\
(5.22)\end{array}$ & $\begin{array}{l}5.46 \\
(7.28)\end{array}$ & $\begin{array}{l}-4.67 \\
(7.89)\end{array}$ & $\begin{array}{l}0.05 \\
(4.17)\end{array}$ & & & $0.01 *$ & $0.04 *$ \\
\hline \multicolumn{9}{|l|}{ Ankle moment } \\
\hline $\begin{array}{l}\text { Peak dorsiflexion in loading } \\
\text { response }(\mathrm{Nm} / \mathrm{kg})^{\#}\end{array}$ & $\begin{array}{l}0.00 \\
(0.07)\end{array}$ & $\begin{array}{l}-0.07 \\
(0.08)\end{array}$ & $\begin{array}{l}0.00 \\
(0.04)\end{array}$ & $\begin{array}{l}-0.08 \\
(0.07)\end{array}$ & & & $0.001 * *$ & $<0.001 * *$ \\
\hline
\end{tabular}


Peak plantar flexion in stance 0.67 $(\mathrm{Nm} / \mathrm{kg})$

Ankle power

Peak absorption in stance $(\mathrm{W} / \mathrm{kg})$

\section{Peak generation in stance (W/kg)}

Peak generation in stance $(\mathrm{W} / \mathrm{kg})$ $\begin{array}{lll}-0.30 & -0.58 & -0.30\end{array}$

$(0.15)$

$(0.37)$

$(0.29)$

$-0.28$

(0.17)

$0.006^{* *}$

$0.001 * * \quad 0.341$

$\begin{array}{llll}0.22 & 0.32 & 0.24 & 0.20\end{array}$

$\begin{array}{lll}(0.15) & (0.22) \quad(0.22)\end{array}$
0.20

$(0.20) \quad 0.055$

0.623

AFO-OD: AFO with an oil damper; joint angle: dorsiflexion, flexion +; internal joint moment: plantar flexion, extension +; mean (standard deviation) for normally distributed data; median (interquatile range) for non-normally distributed data; ns: not significant $* \mathrm{p}<0.05 ; * * \mathrm{p}<0.01 ;{ }^{\#}$ Not normally distributed 


\section{Figures}
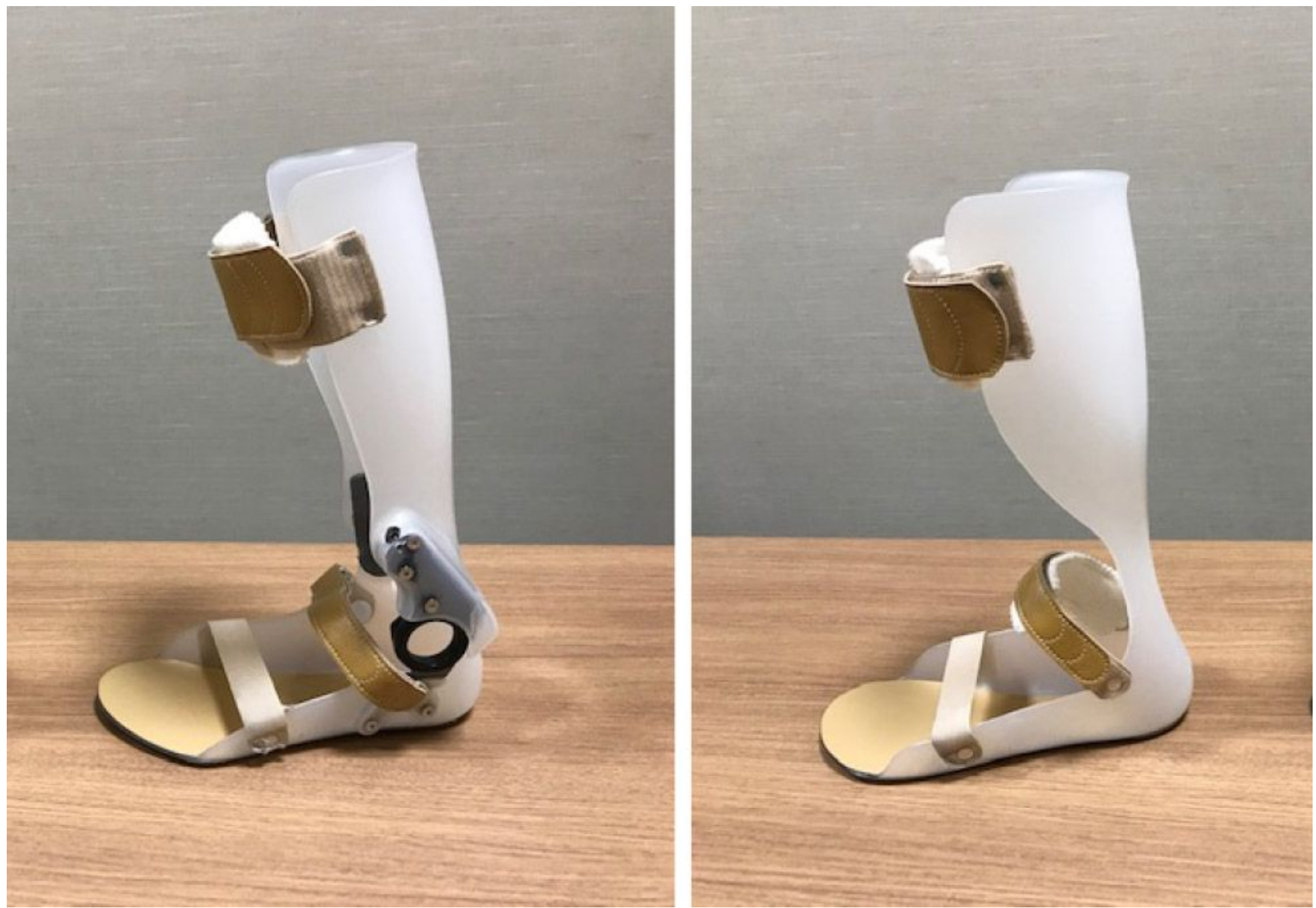

\section{Figure 1}

The ankle-foot orthoses used in this study. Left: An ankle-foot orthosis with an oil damper (AFO-OD). Right: A nonarticulated ankle-foot orthosis. 


\section{Enrollment}

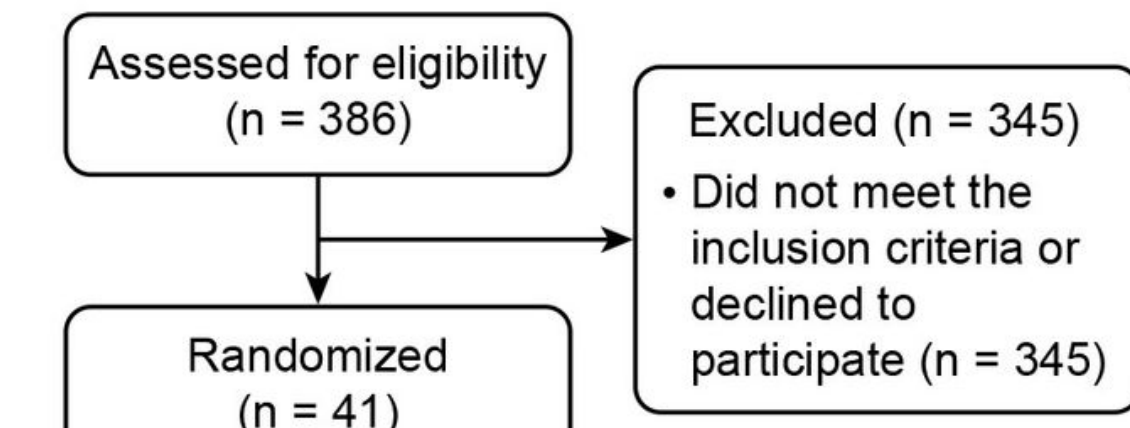

Allocated to AFO-OD

$$
(n=21)
$$

- Received allocated intervention ( $n=21$ )

\section{Allocation}

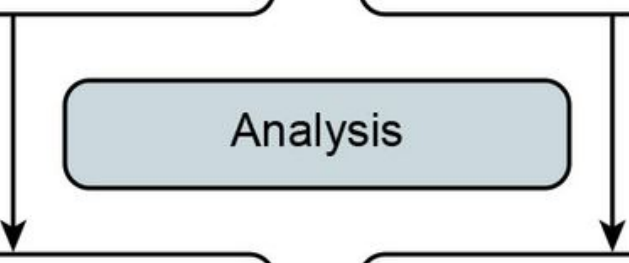

Analyzed $(n=17)$

- Excluded from analysis (missing data) $(n=1)$

- Excluded from analysis (negative step length) $(n=3)$
Analyzed $(n=19)$

- Excluded from analysis (missing data) $(n=1)$

\section{Figure 2}

Consolidated Standard of Reporting Trials (CONSORT) flowchart. 
Without AFO condition
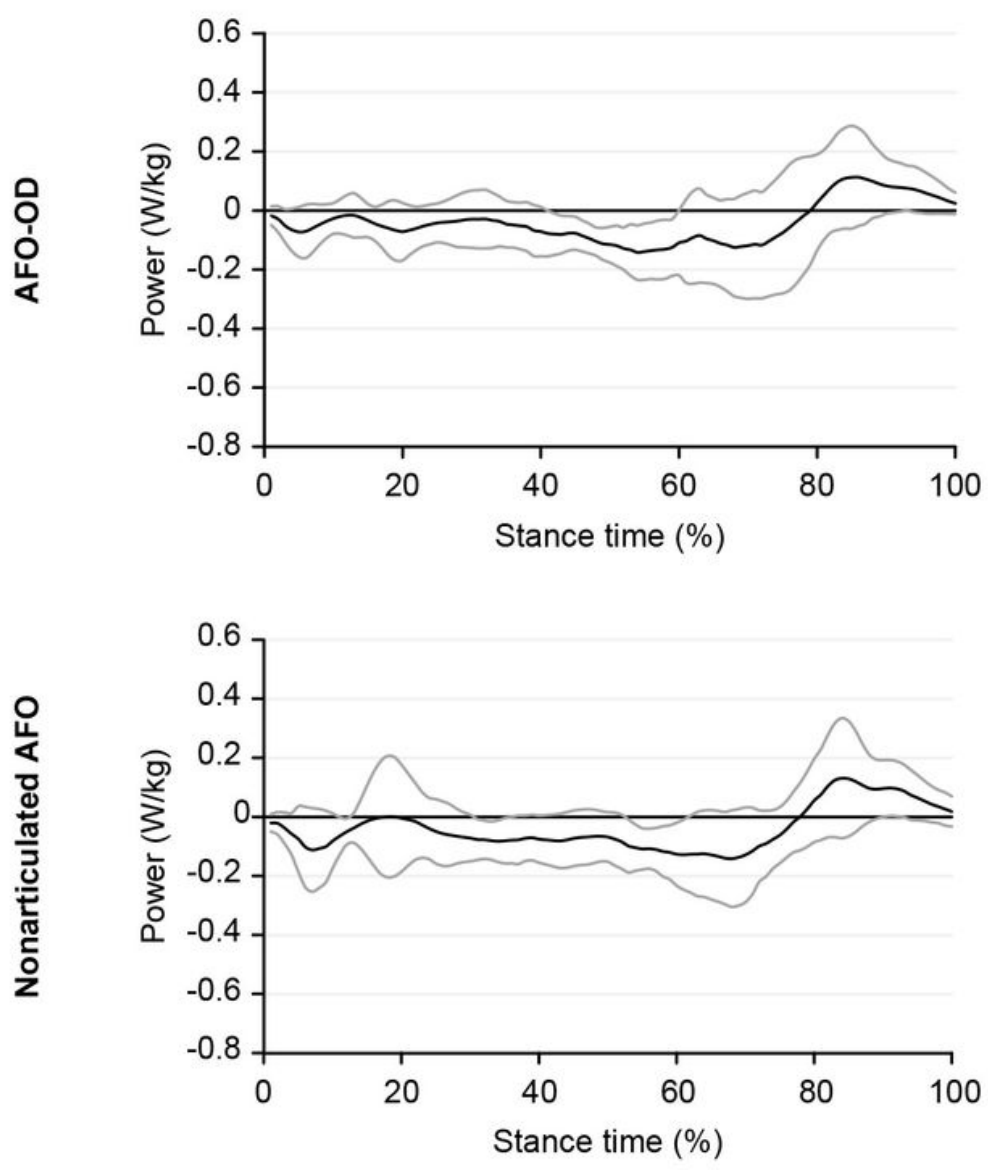

With AFO condition
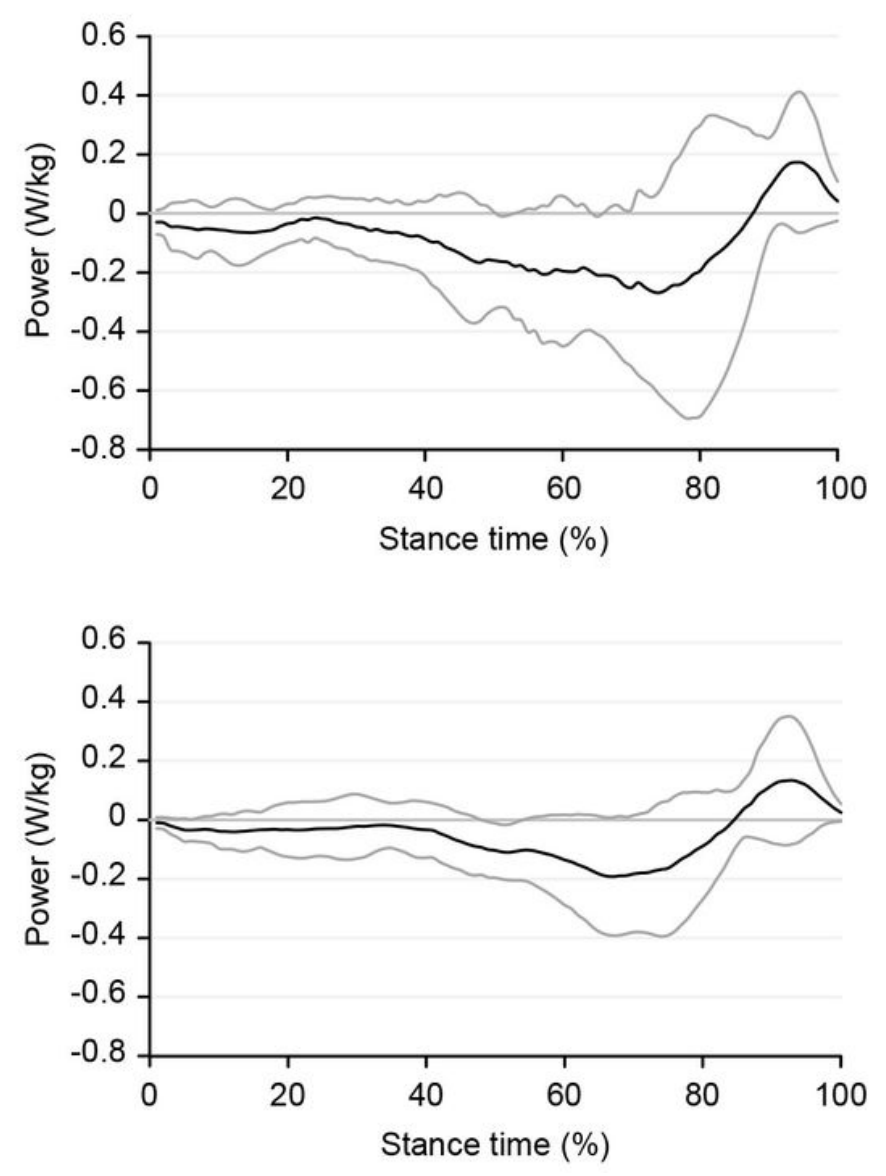

\section{Figure 3}

Graph of average ankle power in paretic stance in each AFO group $(n=17$ in the AFO-OD group, $n=19$ in the nonarticulated group)bold line, average; thin line, standard deviation 


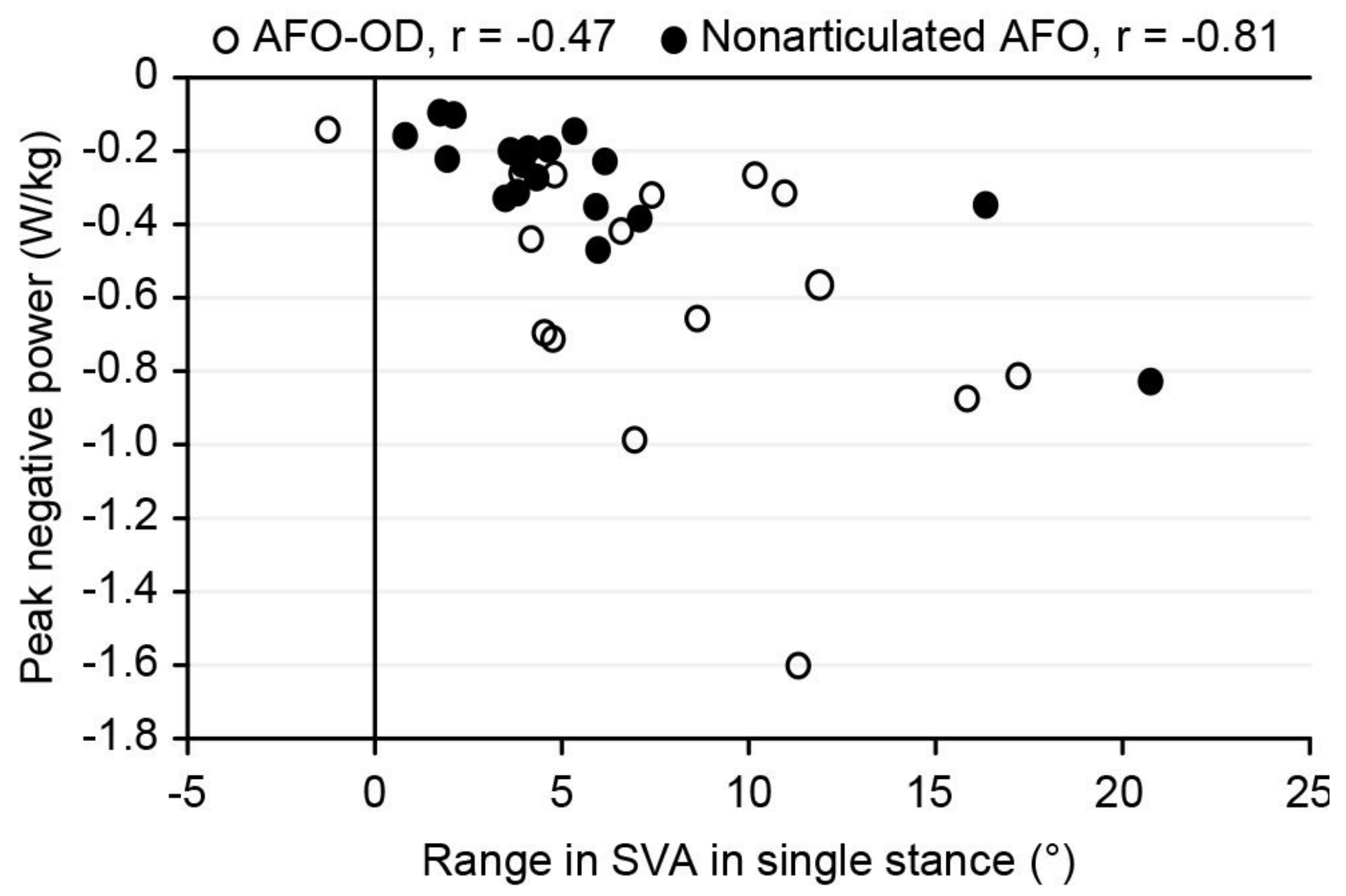

Figure 4

Relationship between shank-to-vertical angle range and ankle peak power absorption. SVA, shank-tovertical angle; AFO-OD, ankle-foot orthosis with oil damper; nonarticulated AFO, nonarticulated ankle-foot orthosis 